Supplement of Hydrol. Earth Syst. Sci., 23, 125-138, 2019

https://doi.org/10.5194/hess-23-125-2019-supplement

(c) Author(s) 2019. This work is distributed under

the Creative Commons Attribution 4.0 License.

(c) (1)

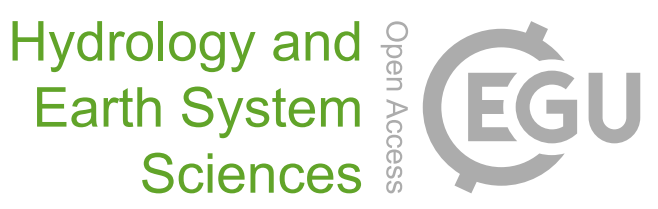

Supplement of

\title{
Understanding variability in root zone storage capacity in boreal regions
}

Tanja de Boer-Euser et al.

Correspondence to: Tanja de Boer-Euser (tanjaeuser@gmail.com)

The copyright of individual parts of the supplement might differ from the CC BY 4.0 License. 


\section{Background on study catchments}

Tables S1 and S2 give an overview of available vegetation and climate characteristics of the study catchments. 


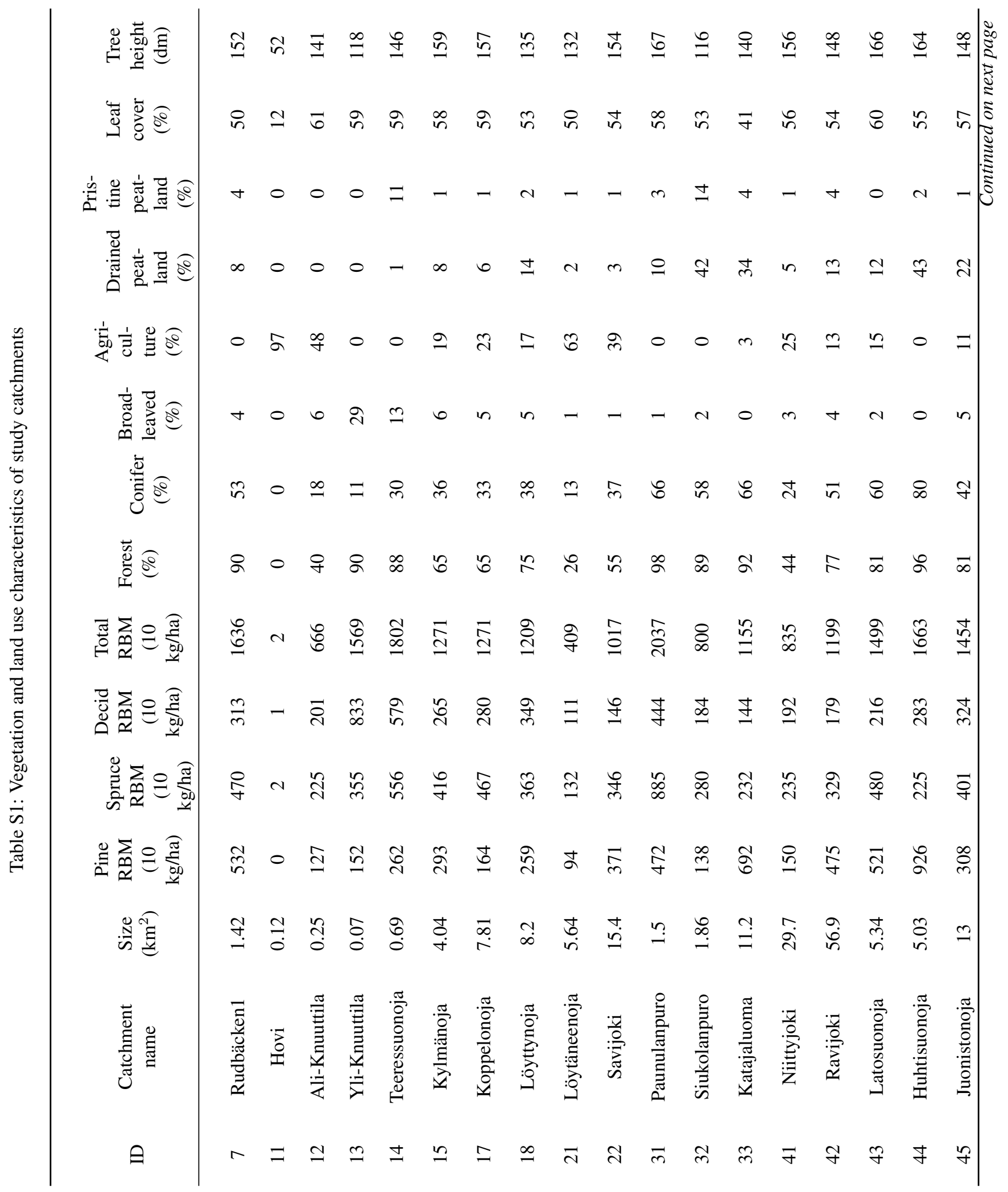




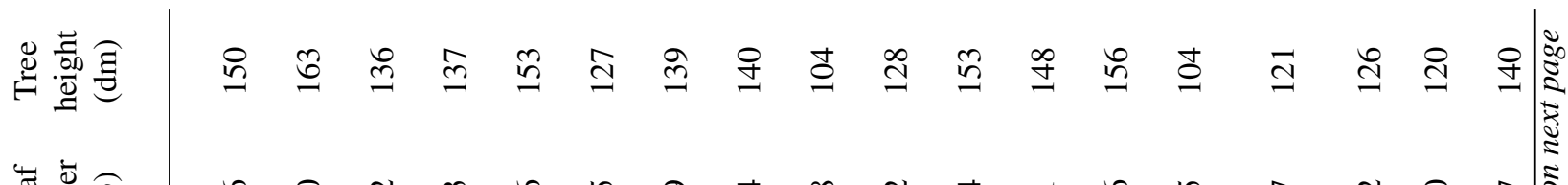

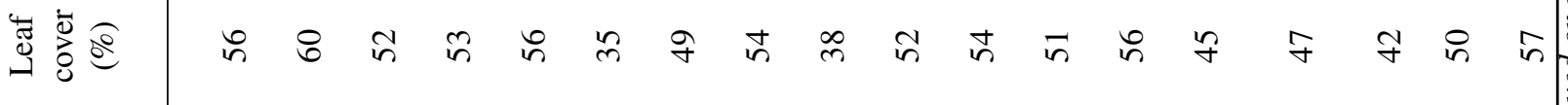

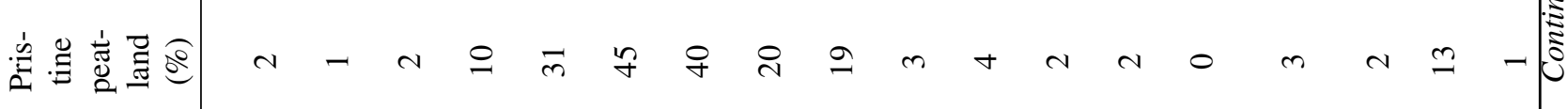

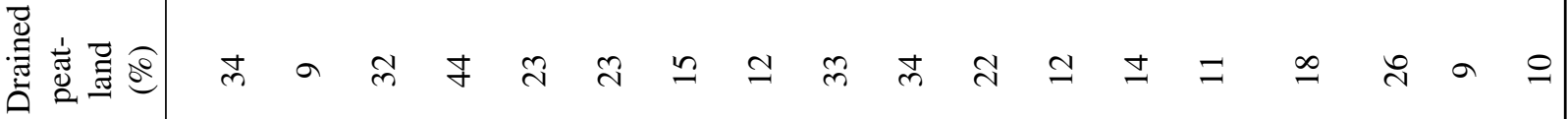

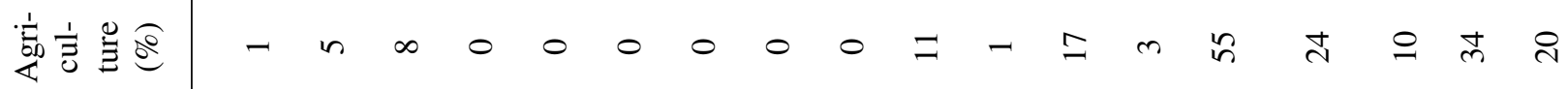

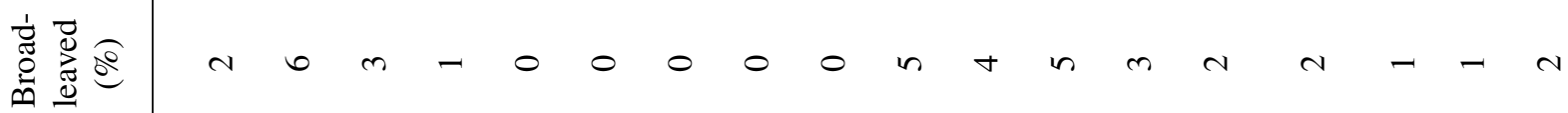

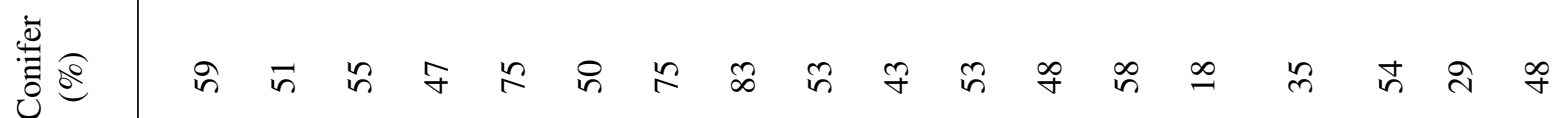

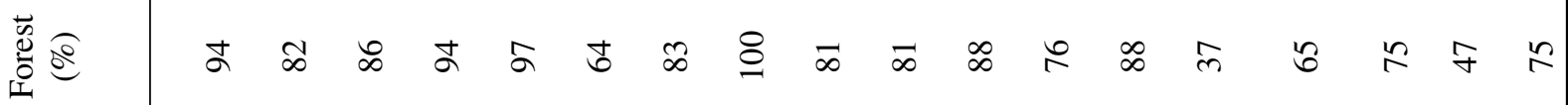

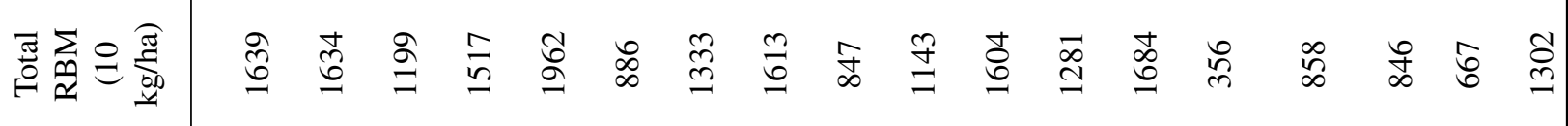

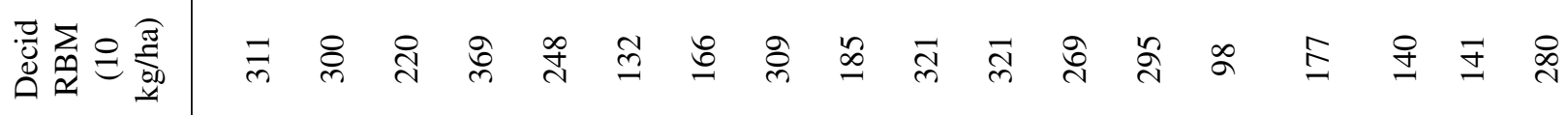

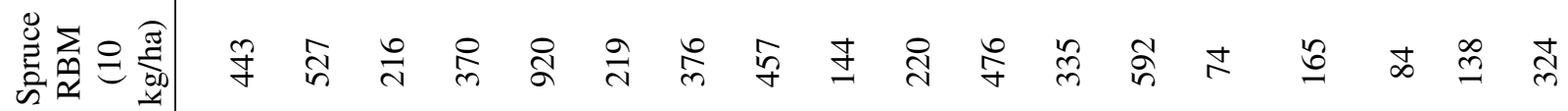

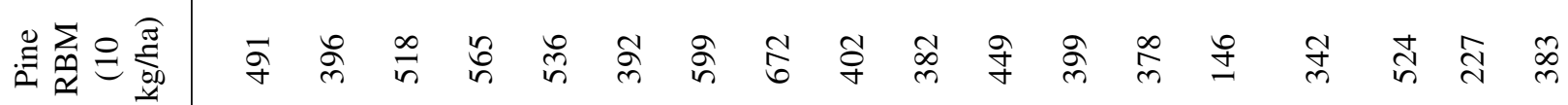

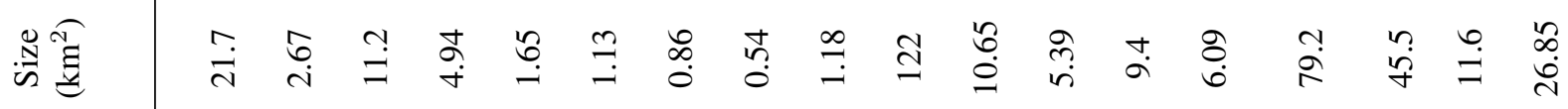

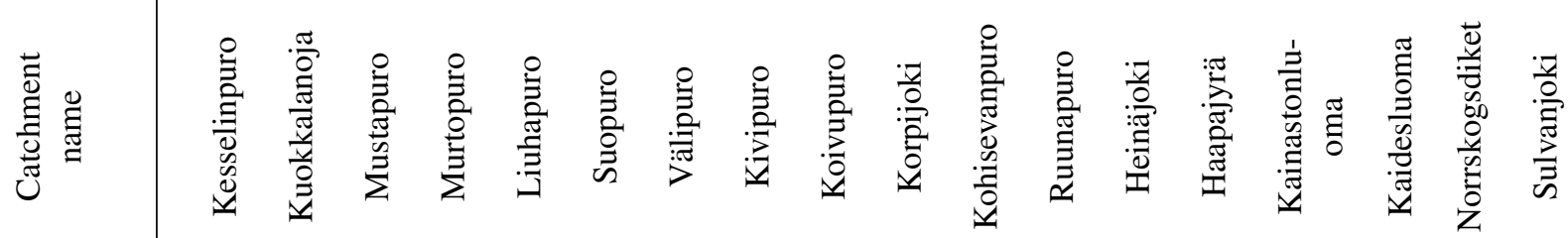

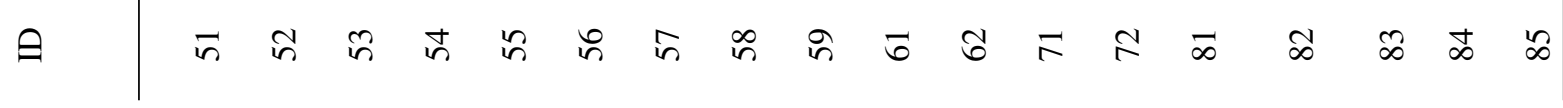




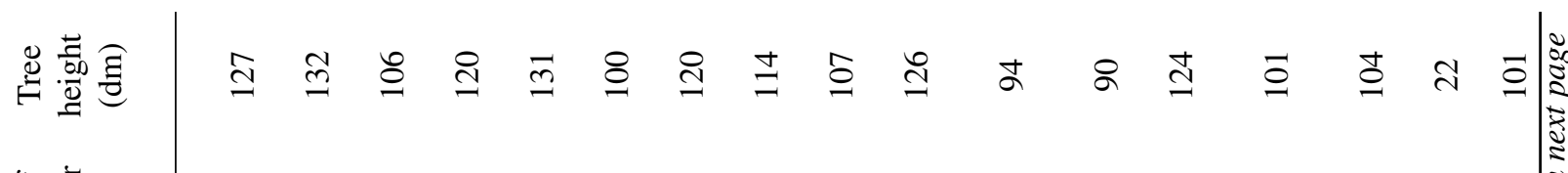

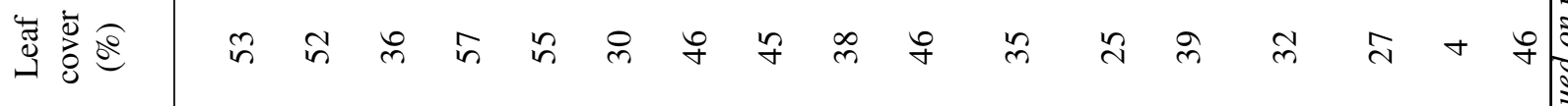

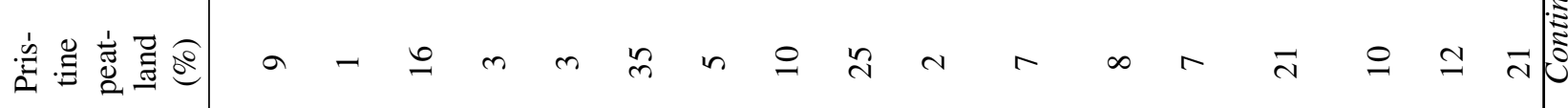

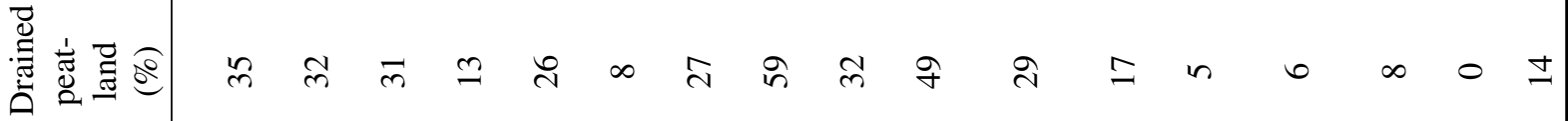

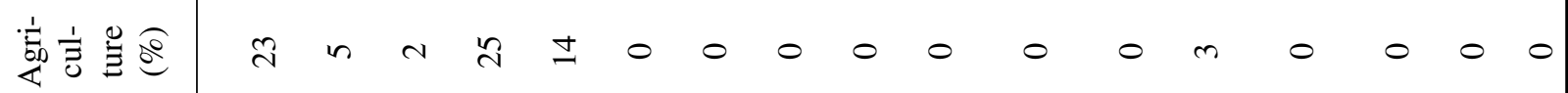

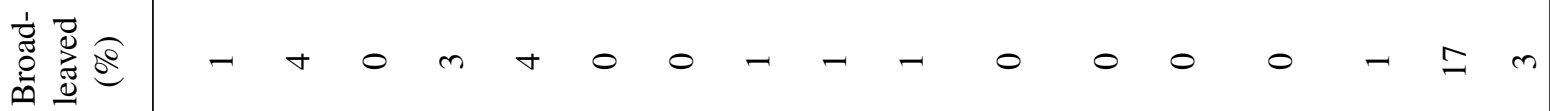

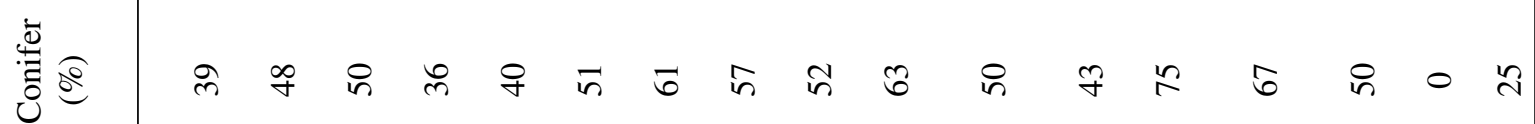

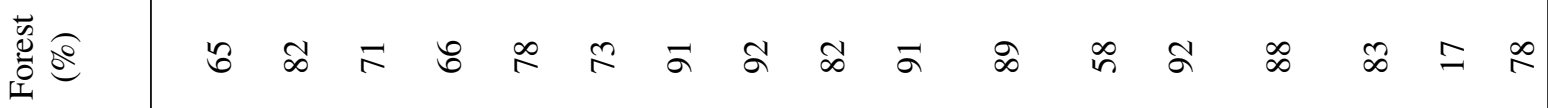

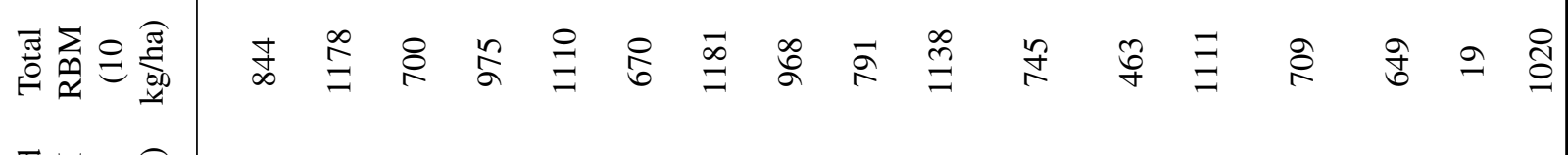

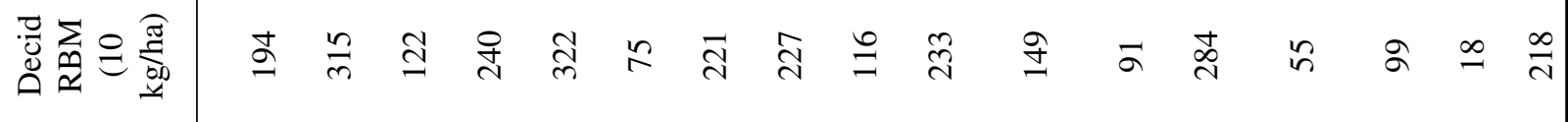

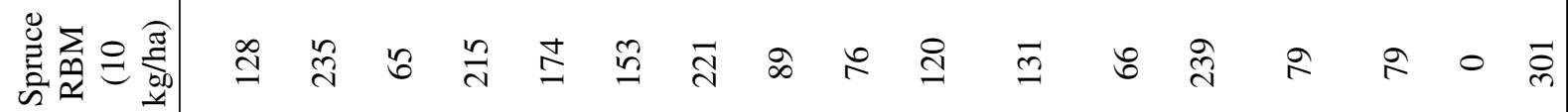

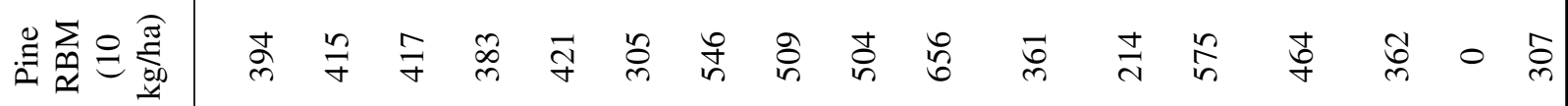

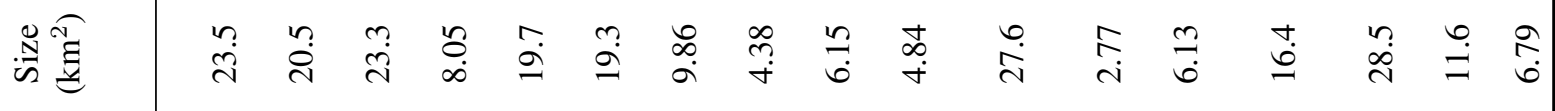

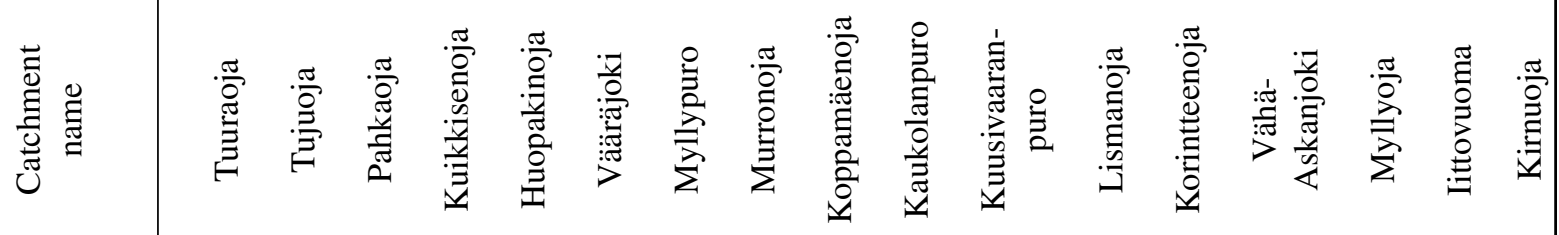
ค न ऽ ๙ 


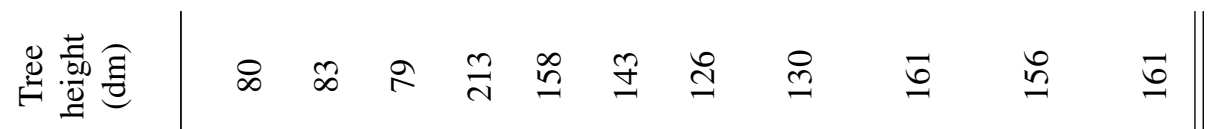

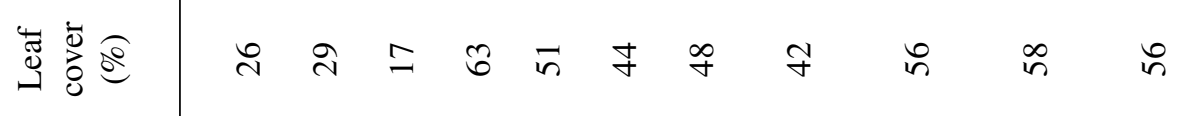

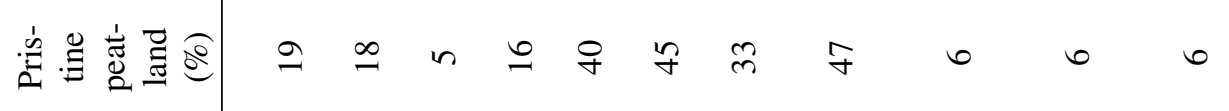

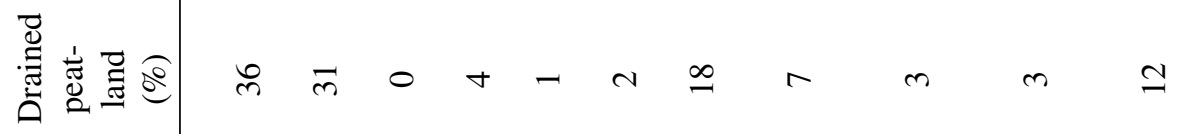

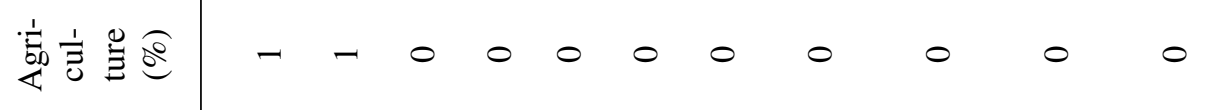

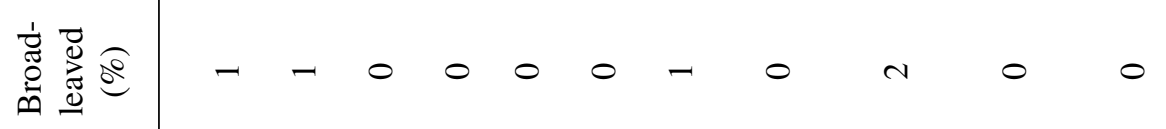

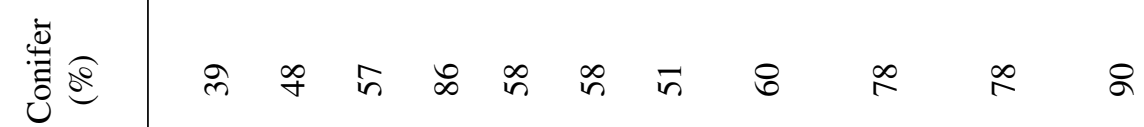

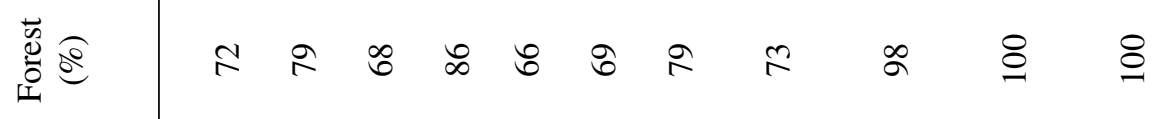

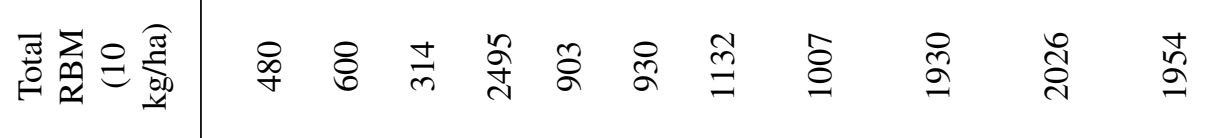

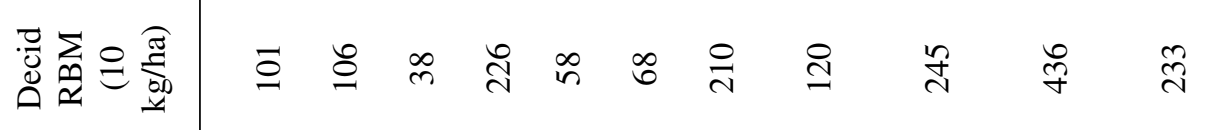

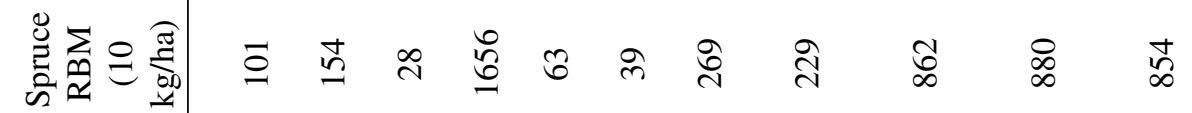

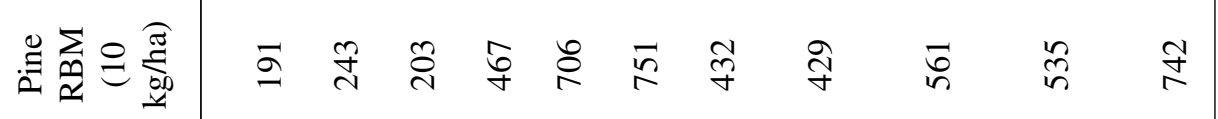

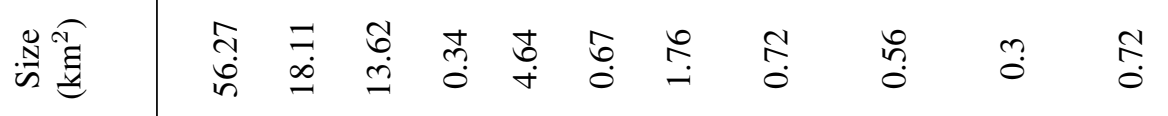

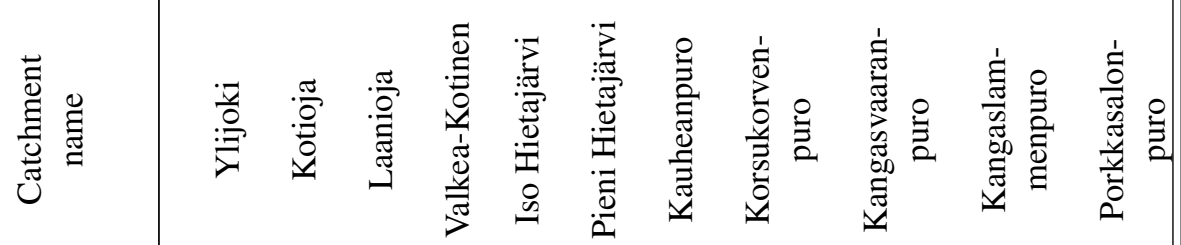

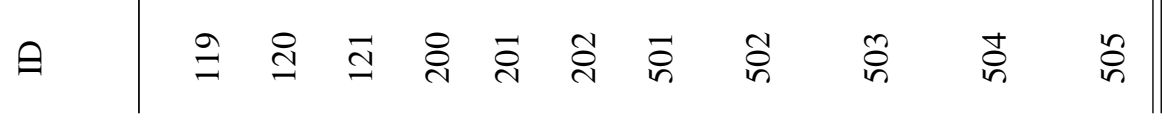


Table S2: Climate characteristics of study catchments

\begin{tabular}{cccccc} 
& Mean & Mean & Max & & \\
& annual & annual & Snow-off \\
tempera- & precipita- & annual & $S_{S W E}$ & $P / E_{P}(-)$ & $\begin{array}{c}\text { (day of } \\
\text { the year) }\end{array}$ \\
& $\left({ }^{\circ} \mathrm{C}\right)$ & $\left.\mathrm{yr}^{-1}\right)$ & $(\mathrm{mm})$ & & \\
\hline
\end{tabular}

\begin{tabular}{|c|c|c|c|c|c|c|}
\hline 7 & Rudbäcken1 & 5 & 682 & 79 & 1.49 & 110 \\
\hline 11 & Hovi & 4.8 & 652 & 79 & 1.42 & 113 \\
\hline 12 & Ali-Knuuttila & 4.8 & 652 & 79 & 1.42 & 113 \\
\hline 13 & Yli-Knuuttila & 4.8 & 652 & 79 & 1.42 & 113 \\
\hline 14 & Teeressuonoja & 4.8 & 652 & 79 & 1.42 & 113 \\
\hline 15 & Kylmänoja & 4.8 & 652 & 79 & 1.42 & 113 \\
\hline 17 & Koppelonoja & 4 & 616 & 65 & 1.41 & 108 \\
\hline 18 & Löyttynoja & 4 & 614 & 65 & 1.44 & 108 \\
\hline 21 & Löytäneenoja & 4.5 & 566 & 73 & 1.09 & 111 \\
\hline 22 & Savijoki & 4.9 & 664 & 73 & 1.28 & 111 \\
\hline 31 & Paunulanpuro & 3.8 & 624 & 117 & 1.5 & 117 \\
\hline 32 & Siukolanpuro & 3.8 & 624 & 117 & 1.5 & 117 \\
\hline 33 & Katajaluoma & 3.9 & 678 & 73 & 1.61 & 111 \\
\hline 41 & Niittyjoki & 4.4 & 646 & 96 & 1.38 & 111 \\
\hline 42 & Ravijoki & 4.4 & 695 & 99 & 1.47 & 113.5 \\
\hline 43 & Latosuonoja & 3.8 & 623 & 107 & 1.49 & 117 \\
\hline 44 & Huhtisuonoja & 3.8 & 623 & 107 & 1.49 & 117 \\
\hline 45 & Juonistonoja & 3.4 & 584 & 97 & 1.43 & 118 \\
\hline 51 & Kesselinpuro & 2.9 & 605 & 132 & 1.32 & 121 \\
\hline 52 & Kuokkalanoja & 2.8 & 645 & 132 & 1.42 & 121 \\
\hline 53 & Mustapuro & 2.7 & 620 & 132 & 1.36 & 121 \\
\hline
\end{tabular}




\begin{tabular}{|c|c|c|c|c|c|c|}
\hline ID & Catchment name & $\begin{array}{c}\text { Mean } \\
\text { annual } \\
\text { tempera- } \\
\text { ture } \\
\left({ }^{\circ} \mathrm{C}\right)\end{array}$ & $\begin{array}{c}\text { Mean } \\
\text { annual } \\
\text { precipita- } \\
\text { tion (mm } \\
\left.\mathrm{yr}^{-1}\right)\end{array}$ & $\begin{array}{c}\text { Max } \\
\text { annual } \\
S_{S W E} \\
(\mathrm{~mm})\end{array}$ & $P / E_{P}(-)$ & $\begin{array}{c}\text { Snow-off } \\
\text { (day of } \\
\text { the year) }\end{array}$ \\
\hline 54 & Murtopuro & 1.7 & 658 & 196 & 1.74 & 127.5 \\
\hline 55 & Liuhapuro & 2 & 624 & 196 & 1.63 & 127.5 \\
\hline 56 & Suopuro & 1.8 & 642 & 196 & 1.71 & 127.5 \\
\hline 57 & Välipuro & 1.8 & 642 & 196 & 1.71 & 127.5 \\
\hline 58 & Kivipuro & 1.8 & 642 & 196 & 1.71 & 127.5 \\
\hline 59 & Koivupuro & 1.8 & 642 & 196 & 1.71 & 127.5 \\
\hline 61 & Korpijoki & 2.4 & 574 & 172 & 1.25 & 125 \\
\hline 62 & Kohisevanpuro & 3 & 593 & 121 & 1.23 & 120 \\
\hline 71 & Ruunapuro & 3.1 & 605 & 119 & 1.25 & 120 \\
\hline 72 & Heinäjoki & 3.5 & 659 & 141 & 1.28 & 121.5 \\
\hline 81 & Haapajyrä & 3.7 & 533 & 78 & 1 & 114 \\
\hline 82 & Kainastonluoma & 3.7 & 547 & 78 & 1.05 & 114 \\
\hline 83 & Kaidesluoma & 3.1 & 545 & 78 & 1.03 & 114 \\
\hline 84 & Norrskogsdiket & 4 & 572 & 75 & 1.13 & 111 \\
\hline 85 & Sulvanjoki & 3.9 & 535 & 78 & 1.06 & 114 \\
\hline 91 & Tuuraoja & 2.8 & 478 & 93 & 1.02 & 117 \\
\hline 92 & Tujuoja & 2.5 & 533 & 112 & 1.09 & 117.5 \\
\hline 93 & Pahkaoja & 2.6 & 575 & 109 & 1.15 & 118 \\
\hline 94 & Kuikkisenoja & 3.3 & 512 & 109 & 1.08 & 118 \\
\hline 101 & Huopakinoja & 2.5 & 514 & 93 & 1.23 & 117 \\
\hline 102 & Vääräjoki & 0 & 581 & 194 & 2.02 & 138 \\
\hline 103 & Myllypuro & 1.3 & 600 & 179 & 1.96 & 133 \\
\hline
\end{tabular}




\begin{tabular}{|c|c|c|c|c|c|c|}
\hline ID & Catchment name & $\begin{array}{c}\text { Mean } \\
\text { annual } \\
\text { tempera- } \\
\text { ture } \\
\left({ }^{\circ} \mathrm{C}\right)\end{array}$ & $\begin{array}{c}\text { Mean } \\
\text { annual } \\
\text { precipita- } \\
\text { tion }(\mathrm{mm} \\
\left.\mathrm{yr}^{-1}\right)\end{array}$ & $\begin{array}{c}\text { Max } \\
\text { annual } \\
S_{S W E} \\
(\mathrm{~mm})\end{array}$ & $P / E_{P}(-)$ & $\begin{array}{l}\text { Snow-off } \\
\text { (day of } \\
\text { the year) }\end{array}$ \\
\hline 104 & Murronoja & 1.9 & 607 & 172 & 1.33 & 125 \\
\hline 105 & Koppamäenoja & 1.9 & 607 & 172 & 1.33 & 125 \\
\hline 106 & Kaukolanpuro & 1.9 & 607 & 172 & 1.33 & 125 \\
\hline 111 & Kuusivaaranpuro & 0 & 498 & 163 & 1.76 & 137 \\
\hline 112 & Lismanoja & -0.6 & 541 & 176 & 1.59 & 139 \\
\hline 113 & Korintteenoja & 0.4 & 552 & 177 & 1.65 & 133 \\
\hline 114 & Vähä-Askanjoki & 0.1 & 546 & 163 & 1.93 & 137 \\
\hline 116 & Myllyoja & -0.6 & 550 & 219 & 1.62 & 144 \\
\hline 117 & Iittovuoma & -2.2 & 434 & 154 & 2.42 & 140 \\
\hline 118 & Kirnuoja & 2 & 494 & 157 & 1.3 & 128 \\
\hline 119 & Ylijoki & 0.7 & 614 & 185 & 1.83 & 135.5 \\
\hline 120 & Kotioja & 0.7 & 614 & 185 & 1.83 & 135.5 \\
\hline 121 & Laanioja & -1.2 & 541 & 207 & 1.95 & 147 \\
\hline 200 & Valkea-Kotinen & 3.7 & 632 & 65 & 1.32 & 108 \\
\hline 201 & Iso Hietajärvi & 2 & 652 & 175 & 1.31 & 130 \\
\hline 202 & Pieni Hietajärvi & 2 & 652 & 175 & 1.31 & 130 \\
\hline 501 & Kauheanpuro & 1.8 & 642 & 196 & 1.71 & 127.5 \\
\hline 502 & Korsukorvenpuro & 1.8 & 642 & 196 & 1.71 & 127.5 \\
\hline 503 & Kangasvaaranpuro & 1.8 & 640 & 196 & 1.68 & 127.5 \\
\hline 504 & Kangaslammenpuro & 1.8 & 640 & 196 & 1.68 & 127.5 \\
\hline 505 & Porkkasalonpuro & 1.8 & 653 & 196 & 1.72 & 127.5 \\
\hline
\end{tabular}


Table S3. Summary of principal component analysis (PCA). The highest loads for each characteristic are shown in bold.

\begin{tabular}{lcc}
\hline & PC1 & PC2 \\
\hline Eigenvalue & 8.20 & 4.68 \\
\% Explained & 34 & 20 \\
Cumulative \% explained & 34 & 54 \\
\hline Forest & -0.364 & $\mathbf{- 0 . 8 2 5}$ \\
Conifer & -0.516 & $\mathbf{- 0 . 8 6 6}$ \\
Broadleaved & $\mathbf{0 . 3 5 0}$ & 0.169 \\
Peatland & $\mathbf{- 0 . 7 2 7}$ & 0.253 \\
Agriculture & $\mathbf{0 . 7 9 6}$ & 0.509 \\
Precipitation & 0.254 & $\mathbf{- 0 . 9 4 4}$ \\
Summer precipitation & 0.021 & $\mathbf{- 0 . 8 6 5}$ \\
max $S_{S W E}$ & $\mathbf{- 1 . 1 6 8}$ & -0.156 \\
$P / E_{p}$ & $\mathbf{- 0 . 9 2 3}$ & -0.068 \\
Longitude & $\mathbf{- 0 . 7 7 1}$ & -0.643 \\
Latitude & $\mathbf{- 1 . 0 8 6}$ & 0.544 \\
Leaf cover & 0.025 & $\mathbf{- 0 . 9 7 4}$ \\
Pine RBM & 0.227 & $\mathbf{0 . 5 6 3}$ \\
Spruce RBM & 0.365 & $\mathbf{0 . 4 3 3}$ \\
Decidious RBM & 0.294 & $\mathbf{0 . 5 3 4}$ \\
Total RBM & 0.298 & $\mathbf{0 . 5 2 5}$ \\
Tree height & 0.528 & $\mathbf{- 0 . 9 6 1}$ \\
Drained peat & $\mathbf{- 0 . 1 9 8}$ & -0.029 \\
Pristine peat & $\mathbf{- 0 . 7 5 1}$ & -0.232 \\
Timing max $S_{S W E}$ & $\mathbf{- 1 . 2 2 1}$ & 0.071 \\
snow-off & $\mathbf{- 1 . 2 0 6}$ & 0.237 \\
mean annual temperature & $\mathbf{1 . 1 8 6}$ & -0.349 \\
Tdemand & $\mathbf{0 . 8 5 7}$ & -0.495 \\
Gap $S_{S W E} E_{p}$ & $\mathbf{- 1 . 1 0 0}$ & -0.041 \\
\hline & & \\
\hline
\end{tabular}

\section{Background on correlations between catchment characteristics}

\subsection{Principal component analysis}

Table S3 shows the explained variance of the first two principal components, together with the loadings of all used catchment characteristics on these two principal components.

\section{$5 \quad 2.2$ Correlation matrix}

Figure S1 shows the correlations between $S_{r}$ and the various catchment characteristics. From this figure it follows that the strongest positive correlation was found between $S_{r}$ and the mean annual temperature and the strongest negative correlation was found for $S_{r}$ and the (timing of) maximum $S_{S W E}$. Further, it can be seen that a strong correlation exits between the different vegetation characteristics and between the different climate variables. In addition, the different land covers (except for drained peatlands) also show a significant correlation with the climate variables. 


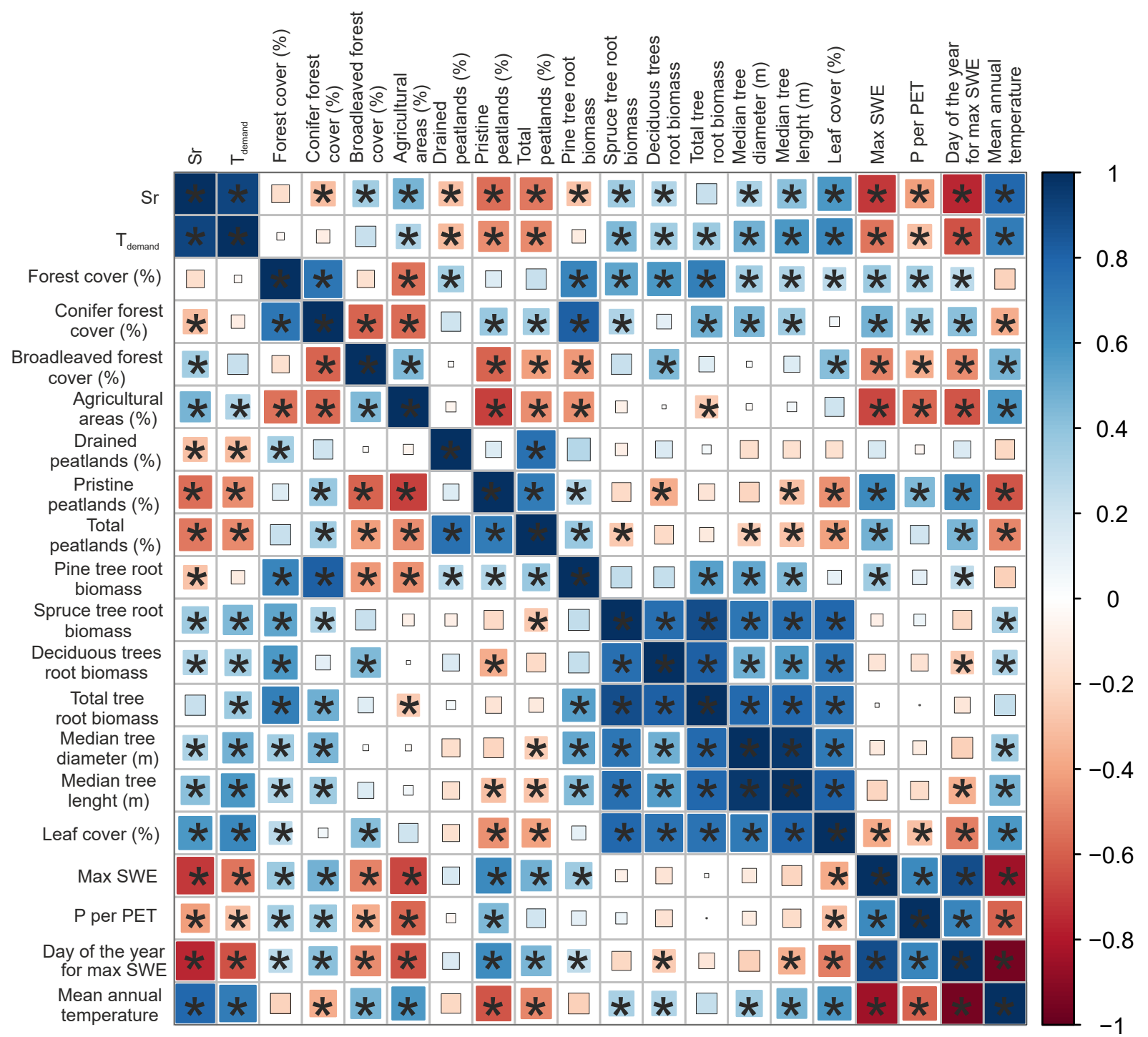

Figure S1. Correlation matrix for calculated root zone storage capacity (20 year return period), calculated transpiration demands (used in the $S_{r}$ calculation) and catchment characteristics. The sizes of the boxes indicate the p-values; the asterisks indicates a significant correlation $(\mathrm{p}<0.05)$. 